\title{
Quantum transport theory for nanostructures with Rashba spin-orbital interaction
}

\author{
Qing-feng Sun* \\ Department of Physics, The University of Hong Kong, Pokfulam Road, Hong Kong, China \\ and Beijing National Laboratory for Condensed Matter Physics and Institute of Physics, Chinese Academy of Sciences, Beijing 100080,
}

China

Jian Wang

Department of Physics, The University of Hong Kong, Pokfulam Road, Hong Kong, China

Hong Guo

Center for the Physics of Materials and Department of Physics, McGill University, Montreal, Quebec, Canada H3A 2T8

(Received 17 November 2004; revised manuscript received 31 January 2005; published 14 April 2005)

\begin{abstract}
We report on a general theory for analyzing quantum transport through devices in the metal-QD-metal configuration where QD is a quantum dot or the device-scattering region which contains Rashba spin-orbital and electron-electron interactions. The metal leads may or may not be ferromagnetic, and they are assumed to weakly couple to the QD region. Our theory is formulated by second quantizing the Rashba spin-orbital interaction in spectral space (instead of real space), and quantum transport is then analyzed within the Keldysh nonequilibrium Green's function formalism. The Rashba interaction causes two main effects to the Hamiltonian: (i) it gives rise to an extra spin-dependent phase factor in the coupling matrix elements between the leads and the QD, and (ii) it gives rise to an interlevel spin-flip term, but forbids any intralevel spin flips. Our formalism provides a starting point for analyzing many quantum transport issues where spin-orbital effects are important. As an example, we investigate the transport properties of a Aharnov-Bohm ring in which a QD having a Rashba spin-orbital and electron-electron interactions is located in one arm of the ring. A substantial spin-polarized conductance or current emerges in this device due to the combined effect of a magnetic flux and the Rashba interaction. The direction and strength of the spin polarization are shown to be controllable by both the magnetic flux and a gate voltage.
\end{abstract}

DOI: 10.1103/PhysRevB.71.165310 PACS number(s): 73.23.-b, 72.25.Dc, 85.75. - d, 73.40.Sx

\section{INTRODUCTION}

The spin-orbit (SO) interaction in semiconductors has attracted great attention in recent years ${ }^{1-12}$ as it plays a very interesting role in the emerging field of semiconductor spintronics. SO interaction can couple the spin degree of freedom of an electron to its orbital motion and vice versa, thereby giving a useful handle for manipulating and controlling the electron spin by external electric fields or gate voltages. The $\mathrm{SO}$ is an intrinsic interaction having its origin in relativistic effects, but it is believed to be substantial in some semiconductors. More than ten years ago, Datta and Das theoretically analyzed the possibility of a spin transistor that worked due to the Rashba SO interaction that induced spin precessions in a semiconductor ${ }^{8}$ with ferromagnetic leads. More recently, Murakami et al. ${ }^{4}$ and Sinova et al..$^{5}$ theoretically predicted that a substantial amount of dissipationless quantum spin current could be generated by a co-action of electric field and SO interaction. ${ }^{4-6}$ Shen et al. found a resonant-spin Hall conductance in a two-dimensional (2D) system with Rashba SO interaction under a perpendicular magnetic field. ${ }^{7}$ There are also many other works on related issues where SO interaction plays a central role, ${ }^{13-18}$ and this research direction is expanding by a very rapid pace due to its possible application to spintronics.

A semiconductor spintronic device is likely to be based on mesoscopic systems and nanostructures where electronelectron $(e-e)$ interactions may be strong. Hence it is desir- able to formulate a general quantum transport theory which can handle SO, $e-e$, and other interactions for systems in the metal-QD-metal configuration. Here the "QD" indicates a quantum dot or the device-scattering region where the various interactions exist, while "metal" is the device lead which extends to electron reservoirs far away. The metal leads may or may not be ferromagnetic, but they are weakly coupled to the QD region. In almost all previous theoretical work, the SO interactions are represented by a real-space Hamiltonian in which $e-e$ interactions and strong correlations are usually neglected. Indeed, it is rather difficult, if not impossible, to handle SO together with $e$-e correlations and other interactions in real space for transport problems. In contrast, the most powerful and general theoretical technique for quantum transport in mesoscopic and nanoscopic systems is the Keldysh nonequilibrium Green's function (NEGF) formalism. ${ }^{19}$ NEGF can handle many-body correlations and interactions in a unified fashion, and it is a well-established formalism. ${ }^{19}$ NEGF is typically formulated in momentum space or other spectral space for theoretical and numerical analysis. This means that all interactions need to be formulated in the spectral space. In other words, in order to conveniently apply NEGF theory, one needs to write the SO interactions in a spectral space with second quantization. To the best of our knowledge, we are not aware of a derivation of such a second quantized form for SO interaction.

It is the purpose of this paper to report a general quantum transport theory for metal-QD-metal devices with $\mathrm{SO}$ and $e$ 
- $e$ interactions, based on the NEGF framework. We begin by presenting a pedagogical discussion of the general physics of SO interaction by quantizing the corresponding classical action, which gives a vivid physical picture of SO interaction. We then second quantize the real-space SO interaction Hamiltonian in a proper spectral space, so that it can be analyzed by NEGF for the quantum-transport properties of metal-QD-metal devices. Although the derivations are general, we specialize on a metal-QD-metal device where the QD is described by the Anderson model plus the Rashba SO interaction, and the leads are ferromagnetic metal. The second quantized Hamiltonian can then be analyzed within NEGF and well-established many-body theoretical methods. ${ }^{20-23}$ To illustrate our formalism, we investigate the quantum-transport properties of a Aharnov-Bohm ring, where a QD having Rashba SO and $e-e$ interactions sits in one arm of the ring. We found that a substantial spinpolarized conductance or current emerges in this device when a magnetic flux passes through the ring. In particular, its spin-polarized direction and strength are controllable by both the magnetic flux and a gate voltage, hence the predictions are testable experimentally.

The paper is organized as follows. In the Sec. II we discuss the many-body Hamiltonian of a general metal-QDmetal device structure and present a pedagogical discussion of the SO interactions in real space. We then proceed, in Sec. III, to second quantize the Rashba SO interaction in spectral space, so that the entire device Hamiltonian can be second quantized. This process is divided into several subsections, and careful derivations and discussions are presented. A brief summary of the second-quantized Hamiltonian in spectral space is given in Sec. III F. In Sec. IV, we analyze the quantum-transport properties of a modified $A B$ ring which contains a QD in one arm of the ring, and both Rashba SO and $e$-e interactions exist in the QD. Finally, Sec. V summarizes the results of our work.

\section{HAMILTONIAN OF THE METAL-QD-METAL DEVICE}

In this section we discuss the Hamiltonian of a general device structure in the metal-QD-metal device configuration. By presenting a very useful pedagogical discussion on the classical forces acting on moving charges and spins inside electrical and magnetic fields, we realize that the SO interaction originates from the force (torque) of the electrical field on the moving spin. This allows us to write the Hamiltonian for the SO interaction in real space $H_{\mathrm{so}}(\mathbf{r})$ and in particular we derive the Rashba SO interaction. Of course, the derived $H_{\text {so }}(\mathbf{r})$ is the same as that from the Dirac equation but the pedagogical discussion gives a vivid physical picture of the SO interactions for quantum transport in solid-state devices. In fact, in the literature of relativistic quantum mechanics, such as the book of Bjorken and Drell, ${ }^{24} \mathrm{SO}$ interaction has been discussed with the point of view of quantizing the classical force acting on the moving spin by the external electric field. We found such a pedagogical discussion in the context of solid-state electronics to be very useful.

The general device structure we consider is schematically shown in Fig. 1(a), where the scattering region (QD) is con-
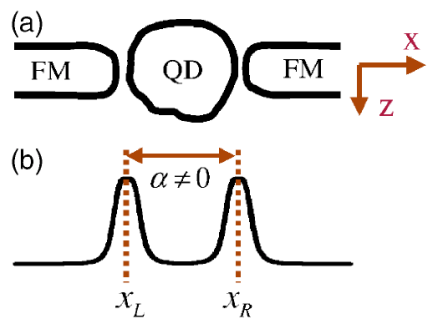

FIG. 1. (Color online) (a) Schematic diagram for a metal-QDmetal device configuration where the QD is weakly coupled to two ferromagnetic leads. (b) Schematic diagram for the scattering potential along the $x$ direction. The Rashba SO interaction is assumed to exist only in the central QD region, i.e., $\alpha=0$ for regions with $x<x_{L}$ and $x>x_{R}$.

nected to the outside world by coupling to two ferromagnetic metal (FM) leads. The permanent magnetic moments of the leads are denoted by the vectors $\mathbf{M}_{\beta}$ where $\beta=L, R$ indicates the left and right leads. The QD is assumed to be weakly coupled to the leads due to the potential barriers at the two metal-QD junctions [Fig. 1(b)]. Inside the QD there are SO and $e$ - $e$ interactions, while these interactions are neglected in the leads. There may also be an external magnetic field $\mathbf{B}(\mathbf{r})$. For this device, the total many-body Hamiltonian can be written as

$$
H\left(\mathbf{r}_{1}, \mathbf{r}_{2}, \ldots, \mathbf{r}_{N}\right)=\sum_{i} H_{s}\left(\mathbf{r}_{i}\right)+\sum_{i, j(i \neq j)} H_{I}\left(\mathbf{r}_{i}, \mathbf{r}_{j}\right),
$$

where the second term is the $e$-e interaction $H_{I}\left(\mathbf{r}_{1}, \mathbf{r}_{2}\right)$ and the first term is from the single-particle Hamiltonian $H_{s}(\mathbf{r})$,

$$
\begin{gathered}
H_{I}\left(\mathbf{r}_{1}, \mathbf{r}_{2}\right)=\frac{e^{2}}{2\left|\mathbf{r}_{1}-\mathbf{r}_{2}\right|^{2}}, \\
H_{s}(\mathbf{r})=\frac{\mathbf{p}^{2}}{2 m^{*}}+V(\mathbf{r})+\hat{\sigma} \cdot \mathbf{M}(\mathbf{r})+\hat{\sigma} \cdot \mathbf{B}(\mathbf{r})+H_{\mathrm{so}}(\mathbf{r}) .
\end{gathered}
$$

$H_{s}$ contains the usual single-particle terms: the kinetic and potential energies, the interaction energy with the magnetic moment $\mathbf{M}$ in the ferromagnetic leads, and the Zeeman energy. ${ }^{25}$ The last term in Eq. (3) is the SO interaction $H_{\mathrm{so}}$. Although the real-space form of $H_{\text {so }}$ is known ${ }^{26,27}$ from the Dirac equation, in the following we present a pedagogical discussion about it.

Transport in our device is about the motion of two entities-charge and spin, in two fields-electric and magnetic fields. Therefore there are a total of four actions due to the fields on the charge and spin: (i) the electric or Coulomb force on the charge; (ii) the Lorentz force on the moving charge; (iii) the magnetic force on the spin (Zeeman); and (iv) the electric force on the moving spin. Of these four actions, (i) -(iii) are well-known and familiar, but (iv) is much less so. Where does (iv) come from? It comes due to a purely relativistic effect. ${ }^{28}$ Consider a spin which produces a magnetic field in the space surrounding it; if this spin is moving, by a relativistic transform we obtain an electric field (in addition to the magnetic field). In other words, a moving spin produces an electric field. Conversely, if a spin is mov- 
ing inside an external electric field, it will be subjected to an action (torque). In this sense, (iv) is the counterpart of the Lorentz force. It has been shown that a moving spin with velocity $\mathbf{v}$ inside an electric field $\mathbf{E}$ is subjected to a torque action with an interactive potential energy, ${ }^{28,29}$

$$
\frac{e \hbar}{4 m_{e} c^{2}} \sigma \bullet(\mathbf{v} \times \mathbf{E}),
$$

where $m_{e}$ is the electron mass, $c$ is the speed of light, and the electron spin is $\mathbf{s}=(\hbar / 2) \sigma$. Of course, this is a classical expression.

To quantize the classical torque energy (4), we make the following replacements: electric field $\mathbf{E} \rightarrow \boldsymbol{\nabla} V(\mathbf{r}) / e$ where $V(\mathbf{r})$ is the potential energy of the system, and the speed $\mathbf{v}$ $\rightarrow \mathbf{p} / m_{e}$ where $\mathbf{p}$ is the momentum operator. The quantummechanical correspondence of expression (4) becomes

$$
\begin{aligned}
& \frac{\hbar}{8 m_{e}^{2} c^{2}}(\hat{\sigma} \bullet[\mathbf{p} \times \boldsymbol{\nabla} V(\mathbf{r})]-\hat{\sigma} \bullet[\boldsymbol{\nabla} V(\mathbf{r}) \times \mathbf{p}])=\frac{\hbar}{8 m_{e}^{2} c^{2}}(\hat{\sigma} \bullet[\mathbf{p} \\
& \quad \times \boldsymbol{\nabla} V(\mathbf{r})]+\boldsymbol{\nabla} V(\mathbf{r}) \cdot(\hat{\sigma} \times \mathbf{p})),
\end{aligned}
$$

where $\hat{\sigma}=\left(\hat{\sigma}_{x}, \hat{\sigma}_{y}, \hat{\sigma}_{z}\right)$ is the vector of the Pauli matrix. Expression (5) is exactly the general form of the SO interaction Hamiltonian, usually derived from the Dirac equation in the low-speed limits. ${ }^{24}$ Therefore, the essence of the SO interaction is simply the action of an external electric field on a moving spin.

If the potential $V(\mathbf{r})$ has spherical symmetry, i.e., $V(\mathbf{r})$ $=V(r)$, we have $\boldsymbol{\nabla} V(\mathbf{r})=(\mathbf{r} / r)(d / d r) V(r)$. Then the general spin-orbit interaction of Eq. (5) reduces to the following familiar form:

$$
H_{\mathrm{so}}=-\frac{1}{2 m_{e}^{2} c^{2}} \frac{1}{r} \frac{d}{d r} V(r) \hat{\mathbf{s}} \cdot \hat{\mathbf{l}},
$$

where the orbital angular momentum operator is $\hat{\mathbf{I}}=\mathbf{r} \times \mathbf{p}$. In fact, Eq. (6) is the well-known Thomas SO coupling.

When our device is made of a two-dimensional electron gas (2DEG) in which the electrons are strongly confined in the $y$ direction by a confining potential $V(y)$, such that $d V / d y \gg(d V / d x),(d V / d z)$, then $\boldsymbol{\nabla} V(\mathbf{r}) \approx \hat{y}(d V / d y)$, and the electric field is almost along the $y$ direction. Furthermore, if $V(y)$ is asymmetric with respect to the reflection point $y=0$, then the matrix element $\langle\Psi(y)|(d / d y) V(y)| \Psi(y)\rangle \neq 0$ where $\Psi(y)$ are the basic bound states in the $y$ direction. Under these conditions, the general SO interaction Eq. (5) reduces to the Rashba SO interaction form, ${ }^{26,27}$

$$
H_{\mathrm{so}}=\frac{\hat{y}}{2 \hbar} \cdot[\alpha(\hat{\sigma} \times \mathbf{p})+(\hat{\sigma} \times \mathbf{p}) \alpha],
$$

where $\quad \alpha \sim\langle\Psi(y)|(d / d y) V(y)| \Psi(y)\rangle \quad$ is the interaction coefficient. ${ }^{30}$ Note that an asymmetrical confining potential in the direction perpendicular to the 2DEG (the $\hat{\mathbf{y}}$ direction) is necessary, otherwise $\alpha=0$, and there would be no Rashba SO interaction. It is worth mentioning that the Rashba SO interaction strength $\alpha$ can be tuned in an experiment by an external electric field or gate voltage, which has already been done in some recent experiments. ${ }^{31-34}$ Finally, if we consider other forms of the potential energy $V(\mathbf{r})$, we obtain other kinds of SO interactions, but the essence of the SO coupling is the interaction of the external electric field on the moving spins.

\section{SECOND QUANTIZATION OF THE DEVICE HAMILTONIAN}

In this section we second quantize the device Hamiltonian (1). The focus is to derive the second quantization of the Rashba SO interaction in a spectral form.

\section{A. Without SO interaction}

The second-quantized form for the Hamiltonian (1) of the metal-QD-metal device with nonmagnetic leads $(\mathbf{M}=0)$, in zero magnetic field $(\mathbf{B}=0)$, and without SO interaction $(\alpha$ $=0$ ) can be approximately written in the standard Anderson model,

$$
H=H_{\mathrm{QD}}+\sum_{\beta=L, R} H_{\beta}+H_{T}
$$

where $H_{\mathrm{QD}}$ is the Hamiltonian for the QD region; $H_{\beta}$ is for the leads and $H_{T}$ is the coupling between the leads and the QD,

$$
\begin{gathered}
H_{\mathrm{QD}}=\sum_{n, s} \epsilon_{n} d_{n s}^{\dagger} d_{n s}+\sum_{n s, m s^{\prime}\left(n s \neq m s^{\prime}\right)} U_{n s, n s^{\prime}} \hat{n}_{n s} \hat{n}_{m s^{\prime}}, \\
H_{\beta}=\sum_{k, s} \epsilon_{k \beta} a_{k \beta s}^{\dagger} a_{k \beta s}, \\
H_{T}=\sum_{k \beta, n, s}\left[t_{k \beta n} a_{k \beta s}^{\dagger} d_{n s}+\text { H.c. }\right] .
\end{gathered}
$$

The quantity $\hat{n}_{n s} \equiv d_{n s}^{\dagger} d_{n s} ; s=\uparrow, \downarrow$ (or + and - ) is the spin index, which also describes the spin states, with $|s\rangle=(1,0)^{T}$ and $(0,1)^{T}$ for the spin-up and spin-down states, respectively. $n$ is quantum number for the eigenstates of the single-particle Hamiltonian $H_{s}$ [Eq. (3)] in the isolated QD region with eigenenergy $\epsilon_{n}=\left\langle n\left|H_{s}\right| n\right\rangle . k \beta$ is the quantum index for lead $\beta$ with eigenenergy $\epsilon_{k \beta}=\left\langle k \beta\left|H_{s}\right| k \beta\right\rangle(\beta=L, R) . t_{k \beta, n}=\left\langle k \beta\left|H_{s}\right| n\right\rangle$ describes the coupling strength between the leads and the QD region. Quantity $U_{n s, n s^{\prime}}=\left\langle n s, m s^{\prime}\left|e^{2} / 2\right| \mathbf{r}_{1}-\left.\mathbf{r}_{2}\right|^{2} \mid m s^{\prime}, n s\right\rangle$ is the matrix element for the two-body $e-e$ interaction. Here the $e$-e interaction in the leads region has been neglected. Note that when $\mathbf{M}=\mathbf{B}=\alpha=0, H_{s}$ does not depend on the Pauli matrix $\hat{\sigma}$; therefore $\left[\hat{\sigma}, H_{s}\right]=0$ and $\epsilon_{n}, \epsilon_{k \beta}$, and $t_{k \beta, n}$ are all independent of the spin index $s$.

Ferromagnetic leads $\mathbf{M}_{\beta} \neq 0$ and $H_{s}$ of Eq. (3) contain a term $\hat{\sigma} \cdot \mathbf{M}_{\beta}$. Let us assume that $\mathbf{M}_{\beta}$ has a constant value in each lead $\beta$ although $\mathbf{M}_{L} \neq \mathbf{M}_{R}$ in general. By calculating the matrix elements $\left\langle k \beta s\left|\hat{\sigma^{\bullet}} \mathbf{M}_{\beta}\right| k^{\prime} \beta s^{\prime}\right\rangle=\delta_{k k^{\prime}}\left(\hat{\sigma} \cdot \mathbf{M}_{\beta}\right)_{s s^{\prime}}$, the second quantization for this term can be easily obtained. $H_{\beta}$ becomes

$$
H_{\beta}=\sum_{k, s} \epsilon_{k \beta} a_{k \beta s}^{\dagger} a_{k \beta s}+\sum_{k}\left(a_{k \beta \uparrow}^{\dagger}, a_{k \beta \downarrow}^{\dagger}\right) \hat{\sigma} \cdot \mathbf{M}_{\beta}\left(\begin{array}{c}
a_{k \beta \uparrow} \\
a_{k \beta \downarrow}
\end{array}\right) .
$$

Due to the existence of $\hat{\sigma} \cdot \mathbf{M}_{\beta}$, the state $|k \beta s\rangle$ is usually not an eigenstate of isolated lead $\beta$, and $\left\langle a_{k \beta s}^{\dagger} a_{k \beta s}\right\rangle$ is not equal to 


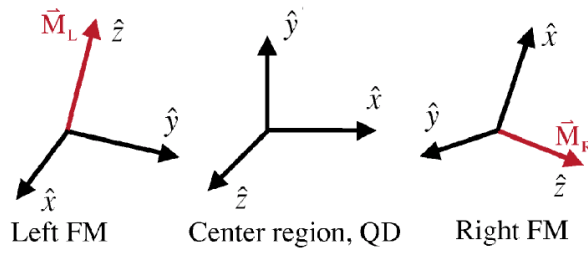

FIG. 2. (Color online) Schematic diagram for the spin coordinates, i.e., the spin-up direction in the left lead, the center region, and the right lead, respectively.

the Fermi distribution function $f_{\beta}\left(\epsilon_{k \beta}\right)$, even in equilibrium. In order to conveniently solve the transport problem, we diagonalize $H_{\beta}$ above by a rotational unitary transformation. This is accomplished by setting

$$
\left(\begin{array}{c}
a_{k \beta \uparrow^{\prime}} \\
a_{k \beta \downarrow^{\prime}}
\end{array}\right)=\left(\begin{array}{c}
\cos \frac{\theta_{\beta}}{2} e^{i \phi_{\beta} / 2} \sin \frac{\theta_{\beta}}{2} e^{-i \phi_{\beta} / 2} \\
-\sin \frac{\theta_{\beta}}{2} e^{i \phi_{\beta} / 2} \cos \frac{\theta_{\beta}}{2} e^{-i \phi_{\beta} / 2}
\end{array}\right)\left(\begin{array}{c}
a_{k \beta \uparrow} \\
a_{k \beta \downarrow}
\end{array}\right),
$$

where $\theta_{\beta}$ and $\phi_{\beta}$ are the directional angles of the FM moment $\mathbf{M}_{\beta}$. Under this rotational transformation, the total second-quantized Hamiltonian of the metal-QD-metal device becomes

$$
\begin{aligned}
& H_{\mathrm{QD}}= \sum_{n, s} \epsilon_{n} d_{n s}^{\dagger} d_{n s}+\sum_{n s, m s^{\prime}\left(n s \neq m s^{\prime}\right)} U_{n s, m s^{\prime}} \hat{n}_{n s} \hat{n}_{m s^{\prime}}, \\
& H_{\beta}=\sum_{k, s}\left(\epsilon_{k \beta}+s M_{\beta}\right) a_{k \beta s}^{\dagger} a_{k \beta s}, \\
& H_{T}=\sum_{k \beta, n, s}\left[t_{k \beta n}\left(\cos \frac{\theta_{\beta}}{2} a_{k \beta s}^{\dagger}-s \sin \frac{\theta_{\beta}}{2} a_{k \beta \bar{s}}^{\dagger}\right)\right. \\
& \\
&\left.\quad \times e^{i s \phi_{\beta} / 2} d_{n s}+\text { H . c. }\right],
\end{aligned}
$$

where $M_{\beta}=\left|\mathbf{M}_{\beta}\right|$. This form of the Hamiltonian has been used before, ${ }^{35}$ but two important comments need to be made: (i) In Hamiltonian (12), the states $|k \beta s\rangle$ are eigenstates of $H_{\beta}$ for isolated leads; hence in equilibrium $\left\langle a_{k \beta s}^{\dagger} a_{k \beta s}\right\rangle=f_{\beta}\left(\epsilon_{k \beta s}\right)$ with $\epsilon_{k \beta s} \equiv \epsilon_{k \beta}+s M_{\beta}$. (ii) After the rotational transformation, the spin-up direction in the left FM lead, the QD, and the right FM lead are all different, although they are all aligned in their local $\hat{\mathbf{z}}$ directions. These local coordinate systems are shown in Fig. 2. In the QD, the spin-up direction is still in the original $\hat{\mathbf{z}}$ axis, but in the left and right FM leads, the spin-up direction (i.e., the local $\hat{\mathbf{z}}$ direction) is aligned with the FM moment $\mathbf{M}_{L / R}$ (see Fig. 2). Although this difference in spin-up alignment is not important when the QD bridging the leads has only a single connection (such as in Fig. 1), it is important if the QD region has double or more connections (such as in Fig. 5).

\section{B. Rashba SO interaction (I)}

In this section and Sec. III C, we second quantize the Rashba SO interaction, which is a major component of this paper. The Rashba SO interaction (7) can be split into two terms,

$$
\begin{aligned}
& \frac{\hat{y}}{2 \hbar} \cdot[\alpha(x)(\hat{\sigma} \times \mathbf{p})+(\hat{\sigma} \times \mathbf{p}) \alpha(x)]=\frac{1}{2 \hbar}\left[\alpha(x) \hat{\sigma}_{z} p_{x}\right. \\
& \left.+\hat{\sigma}_{z} p_{x} \alpha(x)\right]-\frac{\alpha(x) \hat{\sigma}_{x} p_{z}}{\hbar} \equiv H_{R 1}+H_{R 2} .
\end{aligned}
$$

For transport direction along the $\hat{\mathbf{x}}$ axis as shown in Fig. 1, these two terms have some essential differences. The first term $H_{R 1}$ gives rise to a spin precession, ${ }^{8}$ while the second term $H_{R 2}$ does not. In particular, $H_{R 1}$ includes a $\delta$-function factor at the metal-QD contacts $\left(x=x_{L / R}\right.$, see Fig. 1) ${ }^{36,37}$ For this reason it cannot be second quantized by simply calculating the matrix element $\left\langle n s\left|H_{R 1}\right| m s^{\prime}\right\rangle$. To overcome this difficulty, one has to choose a new basis set in the QD. This will be accomplished in this section and the $H_{R 2}$ term will be studied in Sec. III C.

For clarity, the real-space single-particle Hamiltonian considered in this subsection is

$$
H_{s}^{1}(\mathbf{r}) \equiv \frac{p_{x}^{2}+p_{z}^{2}}{2 m^{*}}+V(\mathbf{r})+\hat{\sigma} \cdot \mathbf{M}(\mathbf{r})+H_{R 1} .
$$

This is just Eq. (3), but with only the $H_{R 1}$ part of the SO interactions. We make a unitary transformation with the following unitary matrix:

$$
u(x)=\left\{\begin{array}{lll}
1 & x<x_{L} \\
\exp \left\{-i \hat{\sigma}_{z} \int_{x_{L}}^{x} k_{R}(x) d x\right\} & x_{L}<x<x_{R} \\
\exp \left\{-i \hat{\sigma}_{z} \int_{x_{L}}^{x_{R}} k_{R}(x) d x\right\} & x_{R}<x,
\end{array}\right.
$$

where $k_{R}(x) \equiv \alpha(x) m^{*} / \hbar^{2}$. Here $\alpha(x)$ is permitted to have a dependence on the spatial coordinate $x$ inside the QD, and it is zero outside $\left(x_{R}<x\right.$ or $x<x_{L}$, see Fig. 1$)$. Under this unitary transformation, the original basis functions in the QD region, $|n \uparrow\rangle=\varphi_{n}(\mathbf{r})(1,0)^{T}$ and $|n \downarrow\rangle=\varphi_{n}^{*}(\mathbf{r})(0,1)^{T}$, are transformed to

$$
\begin{aligned}
& |n \uparrow\rangle^{\prime}=u(x)|n \uparrow\rangle=e^{-i \int_{x_{L}}^{x} k_{R}(x) d x} \varphi_{n}(\mathbf{r})\left(\begin{array}{l}
1 \\
0
\end{array}\right), \\
& |n \downarrow\rangle^{\prime}=u(x)|n \downarrow\rangle=e^{+i \int_{x_{L}}^{x} k_{R}(x) d x} \varphi_{n}^{*}(\mathbf{r})\left(\begin{array}{l}
0 \\
1
\end{array}\right) .
\end{aligned}
$$

These new basis functions are used to second quantize (14). After the unitary transformation, $H_{s}^{1}$ of Eq. (14) becomes

$$
H_{s}^{1^{\prime}}=u(x)^{\dagger} H_{s}^{1} u(x)=\frac{p_{x}^{2}+p_{z}^{2}}{2 m^{*}}+V(\mathbf{r})-\frac{\hbar^{2} k_{R}^{2}(x)}{2 m^{*}}+\sigma \cdot \mathbf{M}^{\prime}(\mathbf{r}),
$$

where $\mathbf{M}_{L}^{\prime}=\mathbf{M}_{L}$ and $\left|\mathbf{M}_{R}^{\prime}\right|=\left|\mathbf{M}_{R}\right|$, but the directional angles of $\mathbf{M}_{R}^{\prime}$ are changed to $\left(\theta_{R}, \phi_{R}-2 \phi_{s o}\right)$ with $\phi_{\text {so }} \equiv \int_{x_{L}}^{x_{R}} k_{R}(x) d x$.

The essence of the above unitary transformation is the following.

(i) It is equivalent to choosing a space-dependent spin coordinate, as shown in Fig. 3(a), in which the spin- $\hat{\mathbf{z}}$ direc- 

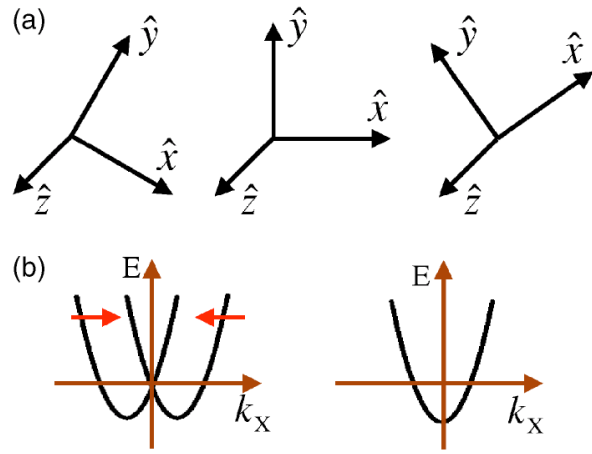

FIG. 3. (Color online) (a) Schematic diagram for the spincoordinate axis in different positions. Here the $x$ and $y$ spin directions are rotated along the $x$ axis in space. (b) Schematic diagram for the dispersion relation before and after the unitary transformation.

tion is fixed everywhere, but the spin- $\hat{\boldsymbol{x}}$ and spin- $\hat{\boldsymbol{y}}$ directions are dependent on the space position $\boldsymbol{r}$. In different positions along the $\boldsymbol{x}$ axis, the directions of the spin- $\hat{x}, \hat{\boldsymbol{y}}$ axis are rotated. In other words, the unitary transform changes us to a rotating frame. It is well known that for an electron moving along the $\boldsymbol{x}$ direction, the Rashba term $H_{R 1}$ gives rise to a spin precession. ${ }^{8,16}$ That is, the spin component in the $x-y$ plane will rotate as the electron moves along the $\hat{\boldsymbol{x}}$ direction, therefore the electron spin is usually not invariant. However, in the rotating frame which follows the spin precession, the spin is invariant; hence $\left[H_{s}^{1^{\prime}}, \hat{\sigma}_{x / y / z}\right]=0$ is satisfied in the QD region.

(ii) The Rashba interaction $H_{R 1}$ can cause an energy split between spin-up and spin-down states for nonzero $k_{x}$, as shown by the energy dispersion in the left panel of Fig. 3(b). ${ }^{21,38}$ The above unitary transformation recovers the alignment of the two dispersion curves, so that the right panel of Fig. 3(b) is obtained. Therefore, after the unitary transformation, the new Hamilton $H_{s}^{1^{\prime}}$ appears to be completely the same as the Hamiltonian without the Rashba interaction $H_{R 1}$, except a rotation of the magnetic moment $\mathbf{M}_{R}$ and a potential energy difference, $-\left[\hbar^{2} k_{R}^{2}(x) / 2 m^{*}\right]=$ $-m^{*} \alpha^{2}(x) /\left(2 \hbar^{2}\right)$, which is a simple constant if $\alpha(x)$ is independent of $x$. Using the same method as that used in Sec. III A, the second quantization of Eq. (18) is easily obtained,

$$
\begin{gathered}
H=H_{\mathrm{QD}}+\sum_{\beta=L, R} H_{\beta}+H_{T}, \\
H_{\mathrm{QD}}=\sum_{n, s} \epsilon_{n} d_{n s}^{\dagger} d_{n s}, \\
H_{\beta}=\sum_{k, s}\left(\epsilon_{k \beta}+s M_{\beta}\right) a_{k \beta s}^{\dagger} a_{k \beta s} . \\
H_{T}=\sum_{k, n, s}\left[t_{k L n}\left(\cos \frac{\theta_{L}}{2} a_{k L s}^{\dagger}-s \sin \frac{\theta_{L}}{2} a_{k L \bar{s}}^{\dagger}\right) e^{i s \phi_{L} / 2} d_{n s}\right. \\
+t_{k R n}\left(\cos \frac{\theta_{R}}{2} a_{k R s}^{\dagger}-s \sin \frac{\theta_{R}}{2} a_{k R \bar{s}}^{\dagger}\right) e^{i s \phi_{R} / 2} e^{-i s \phi_{\mathrm{so}}} d_{n s} \\
+\mathrm{H} . \mathrm{c} .] .
\end{gathered}
$$

This is one of the main results of this paper. The Rashba interaction $H_{R 1}$ gives rise to an extra spin-dependent phase factor $-s \phi_{\text {so }}$ in (20): it is $-\phi_{\text {so }}$ for $s=\uparrow$ and $+\phi_{\text {so }}$ for $s=\downarrow$. Note that the term with this phase factor satisfies the timereversal invariance while $\mathbf{M}_{\beta}=0$, i.e., $\left[T, H_{T}\right]=0$ where $T$ is the time-reversal operator. ${ }^{39}$ This is an expected property because the Rashba SO interaction in real space [Eq. (7)] does satisfy the time-reversal invariance (see the Appendix ). We emphasize that the phase factor $-s \phi_{\text {so }}$ in Eq. (20) is fundamentally different from the phase factor caused by magnetic flux in systems such as the $A B$ ring; the latter is independent of spin $s$, and it destroys timereversal symmetry.

For the special case where $k_{R}(x)=k_{R}=$ const, i.e., independent of coordinate $x$ of the scattering region, we have $\phi_{\text {so }}$ $=k_{R} \times\left(x_{R}-x_{L}\right)$. Then, redefining $e^{-i s k_{R} x_{L}} d_{n s} \rightarrow d_{n s}$, the Hamiltonian (20) can be rewritten in a symmetric manner,

$$
\begin{gathered}
H_{\mathrm{QD}}=\sum_{n, s} \epsilon_{n} d_{n s}^{\dagger} d_{n s}, \\
H_{\beta}=\sum_{k, s}\left(\epsilon_{k \beta}+s M_{\beta}\right) a_{k \beta s}^{\dagger} a_{k \beta s}, \\
H_{T}=\sum_{k, n, s, \beta}\left[t_{k \beta n}\left(\cos \frac{\theta_{\beta}}{2} a_{k \beta s}^{\dagger}-s \sin \frac{\theta_{\beta}}{2} a_{k \beta \bar{s}}^{\dagger}\right)\right. \\
\left.\times e^{i s \phi_{\beta} / 2} e^{-i s k_{R} x_{\beta}} d_{n s}+\text { H.c. }\right] .
\end{gathered}
$$

\section{Rashba SO interaction (II)}

Now we second quantize the second term of the Rashba interaction [Eq. (13)], $H_{R 2} \equiv-\left[\alpha(x) \hat{\sigma}_{x} p_{z} / \hbar\right]$, which can be accomplished by calculating the matrix elements $\left\langle m s^{\prime}\left|u(x)^{\dagger} H_{R 2} u(x)\right| n s\right\rangle=\left\langle m s^{\prime}\left|H_{R 2}^{\prime}\right| n s\right\rangle$. If $s^{\prime}=s$, this matrix element is exactly zero. Hence we only need to calculate the nondiagonal matrix elements, and they are

$$
\begin{aligned}
&\left\langle m \downarrow\left|H_{R 2}^{\prime}\right| n \uparrow\right\rangle= \frac{-\hbar k_{R}}{m^{*}} \int d \mathbf{r} e^{-2 i k_{R^{x}}} \varphi_{m}(\mathbf{r}) p_{z} \varphi_{n}(\mathbf{r}) \equiv t_{m n}^{\mathrm{so}}, \\
&\left\langle n \downarrow\left|H_{R 2}^{\prime}\right| m \uparrow\right\rangle= \frac{-\hbar k_{R}}{m^{*}} \int d \mathbf{r} e^{-2 i k_{R} x} \varphi_{n}(\mathbf{r}) p_{z} \varphi_{m}(\mathbf{r}) \\
&= \frac{\hbar k_{R}}{m^{*}} \int d \mathbf{r} e^{-2 i k_{R} x} \varphi_{m}(\mathbf{r}) p_{z} \varphi_{n}(\mathbf{r})=-t_{m n}^{\mathrm{so}}, \\
&\left\langle n \uparrow\left|H_{R 2}^{\prime}\right| m \downarrow\right\rangle=t_{m n}^{\mathrm{s} *^{*}}, \\
&\left\langle m \uparrow\left|H_{R 2}^{\prime}\right| n \downarrow\right\rangle=-t_{m n}^{\mathrm{so*}} .
\end{aligned}
$$

Here (as well as below) we have assumed $k_{R}(x)$ [or $\alpha(x)$ ] to be independent of $x$, but even if $k_{R}(x)$ depends on $x$, all results are completely the same. With the above matrix ele- 
ments $[(22)-(25)]$, the second quantized form of $H_{R 2}$ is

$$
H_{R 2}=\sum_{m, n(m<n)} t_{m n}^{\mathrm{so}}\left[d_{m \downarrow}^{\dagger} d_{n \uparrow}-d_{n \downarrow}^{\dagger} d_{m \uparrow}\right]+\text { H . c . , }
$$

which can be written in the more compact form,

$$
H_{R 2}=\sum_{m, n} t_{m n}^{\mathrm{so}} d_{m \downarrow}^{\dagger} d_{n \uparrow}+\mathrm{H} . \mathrm{c} .
$$

where it is important to realize that $t_{m n}^{\mathrm{so}}=-t_{n m}^{\mathrm{so}}$. Equation (26) is another main result of this paper.

Some general characteristics of Eq. (26) are, in order.

(i) The property $t_{m n}^{\mathrm{so}}=-t_{n m}^{\mathrm{so}}$ for the matrix elements originates from the time-reversal invariance of the original realspace Rashba Hamiltonian. ${ }^{39}$ Using this property, we can exactly prove that the second-quantized form of $H_{R 2}[\mathrm{Eq}$. (26)] indeed satisfies the time-reversal invariance. ${ }^{39}$

(ii) If $n=m$, we have $t_{n n}^{\mathrm{so}}=-t_{n n}^{\mathrm{so}}$; hence $t_{n n}^{\mathrm{so}}$ must vanish. This means that the Rashba SO interaction cannot induce any intralevel spin flip, i.e., it cannot give rise to a transition $(n \uparrow) \rightarrow(n \downarrow)$ in which the level index $n$ is the same. Therefore, the SO interaction (26) is fundamentally different from that of an external magnetic field. A magnetic field can cause intralevel spin flip, provide a Zeeman energy that relieves spin degeneracy, and induce spin polarization in an isolated QD (see Sec. III E, below).

(iii) The Rashba SO interaction (26) can cause spin flips between different energy levels. This interlevel spin-flip coupling is similar to the intersubband mixing in real space, which has been studied in previous works. ${ }^{13}$ Despite the interlevel spin flips, the system is still at least twofold degenerate for any eigenstates, because $t_{m n}^{\mathrm{so}}=-t_{n m}^{\mathrm{so}}$. This guarantees that at equilibrium an isolated QD has no spin polarization. In the Appendix , the general properties of Eq. (26) are further discussed.

(iv) In fact, because all spin-orbit couplings satisfy timereversal invariance, the above properties and matrix elements $t_{m n}=-t_{n m}$ must hold true in general. In this regard, we note that there exist papers where $t_{m n}=t_{n m}$ and intralevel spin flips are allowed; these effects cannot come from SO interactions, as sometimes claimed.

Let us estimate the value of $t_{n m}^{\mathrm{so}}$. Consider a square QD with linear size $W$. The eigenstates are $\varphi_{n}(\mathbf{r})$ $=(2 / W) \sin \left(n_{x} \pi x / W\right) \sin \left(n_{z} \pi z / W\right)$, hence $t_{m n}^{\text {so }}$ can be easily calculated from Eq. (22). For parameters $W=100 \mathrm{~nm}, \alpha=3$ $\times 10^{-11} \mathrm{eV} \mathrm{m}$ and $m^{*}=0.036 m_{e}$, the intradot level spacing $\Delta \epsilon \approx \hbar^{2} \pi^{2} / 2 m^{*} W^{2} \approx 1 \mathrm{meV}$. This is to be compared with a rough estimate of $\left|t_{m n}^{\mathrm{so}}\right| \sim \hbar^{2} k_{R} / m^{*} W=\alpha / W \sim 0.3 \mathrm{meV}$.

\section{Electron-electron Coulomb interaction}

In order to second quantize the Rashba SO interaction, we have introduced a unitary transformation defined by Eq. (15). Does this transformation affect the familiar second-quantized form of the $e-e$ interaction? Here we show it does not.

Starting from the two-body $e-e$ interaction in real space,

$$
H_{I}\left(\mathbf{r}_{1}, \mathbf{r}_{2}, \ldots \mathbf{r}_{N}\right)=\sum_{i, j(i \neq j)} \frac{e^{2}}{2\left|\mathbf{r}_{i}-\mathbf{r}_{j}\right|^{2}},
$$

we apply the unitary transformation, and the new Hamiltonian $H_{I}^{\prime}$ is

$H_{I}^{\prime}=\sum_{i, j(i \neq j)} u^{\dagger}\left(x_{i}\right) u^{\dagger}\left(x_{j}\right) \frac{e^{2}}{2\left|\mathbf{r}_{i}-\mathbf{r}_{j}\right|^{2}} u\left(x_{j}\right) u\left(x_{i}\right)=\sum_{i, j(i \neq j)} \frac{e^{2}}{2\left|\mathbf{r}_{i}-\mathbf{r}_{j}\right|^{2}}$.

This means $H_{I}^{\prime}=H_{I}$, and the unitary transformation does not affect the form of the $e-e$ interaction. We therefore can directly write the second-quantized $e-e$ interaction in its familiar form,

$$
H_{I}=\sum_{n s, m s^{\prime}\left(n s \neq m s^{\prime}\right)} U_{n s, m s^{\prime}} d_{n s}^{\dagger} d_{n s} d_{m s^{\prime}}^{\dagger} d_{m s^{\prime}},
$$

where the matrix element $U_{n s, m s^{\prime}}$ is

$$
U_{n s, m s^{\prime}}=\left\langle n s, m s^{\prime}\left|\frac{e^{2}}{2\left|\mathbf{r}_{1}-\mathbf{r}_{2}\right|^{2}}\right| m s^{\prime}, n s\right\rangle \text {. }
$$

\section{E. External magnetic field}

The unitary transformations [Eq. (15)] do affect the second-quantized form of the external magnetic fields $\hat{\sigma} \cdot \mathbf{B}$. Consider an arbitrary external magnetic field $\mathbf{B}=\left(B_{x}, B_{y}, B_{z}\right)$, where $B_{x / y / z}$ is projected in the $x / y / z$ direction.

First, we investigate the $z$-direction element $B_{z}$. Under the unitary transformation [Eq. (15)], the term $\hat{\sigma}_{z} B_{z}$ changes to

$$
u^{\dagger}(x) \hat{\sigma}_{z} B_{z} u(x)=e^{i \hat{\sigma}_{z} k_{R} x} \hat{\sigma}_{z} B_{z} e^{-i \hat{\sigma}_{z} k_{R} x}=\hat{\sigma}_{z} B_{z},
$$

which means $\hat{\sigma}_{z} B_{z}$ does not change under the unitary transformation. Therefore its second-quantized form is still

$$
\sum_{n s} s B_{z} d_{n s}^{\dagger} d_{n s}
$$

Second, we investigate the $x$-direction element $B_{x}$, i.e., the term $\hat{\sigma}_{x} B_{x}$ in the Hamiltonian. After the unitary transformation [Eq. (15)], $u^{\dagger}(x)\left(\hat{\sigma}_{x} B_{x}\right) u(x) \neq \hat{\sigma}_{x} B_{x}$, so that it is affected by the transformation. The matrix elements $\left\langle m s^{\prime}\left|u^{\dagger}(x) \hat{\sigma}_{x} B_{x} u(x)\right| n s\right\rangle$ are found to be

$$
\left\langle m \uparrow\left|u^{\dagger} \hat{\sigma}_{x} B_{x} u\right| n \uparrow\right\rangle=\left\langle m \downarrow\left|u^{\dagger} \hat{\sigma}_{x} B_{x} u\right| n \downarrow\right\rangle=0,
$$

$$
\left\langle m \downarrow\left|u^{\dagger} \hat{\sigma}_{x} B_{x} u\right| n \uparrow\right\rangle=\int d \mathbf{r} e^{-i 2 k_{R} x} \varphi_{m}(\mathbf{r}) \varphi_{n}(\mathbf{r}) B_{x} \equiv t_{m n}^{B} B_{x},
$$

$$
\left\langle n \downarrow\left|u^{\dagger} \hat{\sigma}_{x} B_{x} u\right| m \uparrow\right\rangle=t_{m n}^{B} B_{x},
$$

$$
\left\langle n \uparrow\left|u^{\dagger} \hat{\sigma}_{x} B_{x} u\right| m \downarrow\right\rangle=\left\langle m \uparrow\left|u^{\dagger} \hat{\sigma}_{x} B_{x} u\right| n \downarrow\right\rangle=t_{m n}^{B^{*}} B_{x} .
$$

Hence, the second-quantized form of $\hat{\sigma}_{x} B_{x}$ is

$$
\sum_{m, n(n<m)} B_{x} t_{m n}^{B}\left[d_{m \downarrow}^{\dagger} d_{n \uparrow}+d_{n \downarrow}^{\dagger} d_{m \uparrow}\right]+\sum_{n} B_{x} t_{n n}^{B} d_{n \downarrow}^{\dagger} d_{n \uparrow}+\text { H . c . , }
$$

or it can be written in a more compact form, 


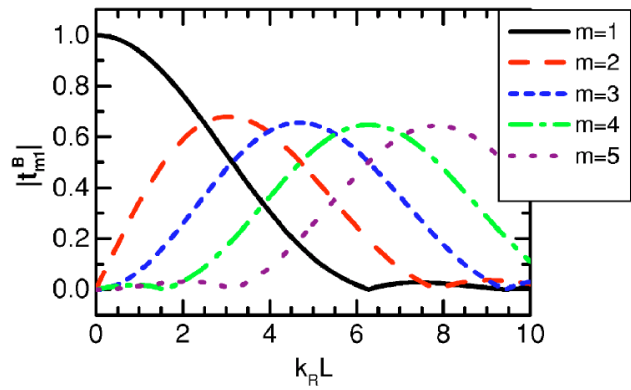

FIG. 4. (Color online) The spin-flip coupling strength $t_{m 1}^{B}$ versus the Rashba SO interaction $k_{R} L$. As $k_{R} L$ increases, more modes $(m)$ are playing a role.

$$
\sum_{m, n} B_{x} t_{m n}^{B} d_{m \downarrow}^{\dagger} d_{n \uparrow}+\text { H. . . , }
$$

with $t_{m n}^{B}=t_{n m}^{B}$. Note that the form of Eq. (33) is very similar to the Rashba term $H_{R 2}$, [Eq. (26)], but there exists an essential difference. Namely, for the magnetic field in the $x$ direction, $t_{m n}^{B}=t_{n m}^{B}$ in Eq. (33), while for the Rashba term, $t_{m n}^{\text {so }}=$ $-t_{n m}^{\text {so }}$ in Eq. (26). We emphasize that this is an essential difference because of two reasons:

(i) The magnetic-field term destroys the time-reversal invariance, it provides a Zeeman energy that breaks the spin degeneracy of the energy levels, and it can induce a spin polarization in equilibrium. In contrast, the Rashba term $H_{R 2}$ satisfies the time-reversal invariance and maintains the two degeneracies.

(ii) When $n=m, t_{n n}^{B}$ can be nonzero so that intralevel spin flips are possible. Furthermore, the $t_{n n}^{B}$ term is usually the largest term in the sum of Eq. (33), e.g., $t_{m n}^{B}=\delta_{m n}$ at $k_{R}=0$. But for the Rashba interaction (26), $t_{n n}^{\mathrm{so}}$ must vanish as discussed before, so that it cannot cause intralevel spin flip. We therefore comment that interactions of the following form, (which have been used in some previous literature):

$$
t d_{\downarrow}^{\dagger} d_{\uparrow}+t d_{\uparrow}^{\dagger} d_{\downarrow}
$$

do not represent the SO interaction. Rather, they describe a magnetic field pointing to the $x$ direction.

In order to estimate the value of $t_{n m}^{B}$, we consider a rectangular QD with length $L$ and width $W . t_{m n}^{B}$ can be obtained as

$$
t_{m n}^{B}=2 \delta_{m_{z}, n_{z}} \int_{0}^{1} d x e^{-2 i k_{R} L x} \sin m_{x} \pi x \sin n_{x} \pi x .
$$

Figure 4 plots the numerical results for $t_{m 1}^{B}$ versus the parameter $k_{R} L$ that is obtained this way. As $k_{R} L$ increases, more $t_{m 1}^{B}$ are in action. If the parameters $\alpha=2 * 10^{-11} \mathrm{eV} \mathrm{m}$ and $L=100 \mathrm{~nm}$, we have $k_{R} L \approx 1$ for $m^{*}=0.036 m_{e}$. For this $k_{R} L$ value, only $t_{11}^{B}$ and $t_{21}^{B}$ are significant.

Finally, the second quantization of the $B_{y}$ term is completely the same as that for the $x$ direction; hence, its secondquantized form is the same as Eq. (33).

\section{F. Brief summary}

Collecting all the pieces of second quantization which we have carried out in this section, for a device in the form of

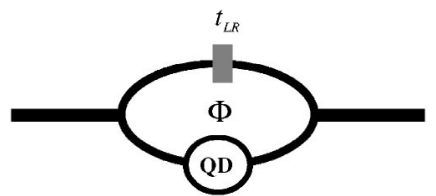

FIG. 5. Schematic diagram for the modified $A B$ ring device. Two normal leads are coupled to the center ring, a magnetic flux threads the ring, and a QD is embedded in one arm of the ring.

metal-QD-metal, where there exists Rashba SO and $e-e$ interactions in the QD, the metal leads are magnetic material, and there exists an external magnetic field B. Hamiltonian (1) becomes

$$
H=H_{\mathrm{QD}}+\sum_{\beta=L, R} H_{\beta}+H_{T},
$$

where

$$
\begin{gathered}
H_{\mathrm{QD}}=\sum_{n, s}\left(\epsilon_{n}+s B_{z}\right) d_{n s}^{\dagger} d_{n s}+\sum_{n s, m s^{\prime}\left(n s \neq m s^{\prime}\right)} U_{n s, m s^{\prime}} \hat{n}_{n s} \hat{n}_{m s^{\prime}} \\
+\sum_{m, n}\left[t_{m n}^{\mathrm{so}} d_{m \downarrow}^{\dagger} d_{n \uparrow}+B_{x} t_{m n}^{B} d_{m \downarrow}^{\dagger} d_{n \uparrow}+\mathrm{H} . \mathrm{c} .\right] \\
H_{\beta}=\sum_{k, s}\left(\epsilon_{k \beta}+s M_{\beta}\right) a_{k \beta s}^{\dagger} a_{k \beta s}, \\
H_{T}=\sum_{k, n, s, \beta}\left[t_{k \beta n}\left(\cos \frac{\theta_{\beta}}{2} a_{k \beta s}^{\dagger}-s \sin \frac{\theta_{\beta}}{2} a_{k \beta \bar{s}}^{\dagger}\right)\right. \\
\left.\times e^{i s \phi_{\beta} / 2} e^{-i s k_{R} x} d_{n s}+\mathrm{H} . \mathrm{c} .\right]
\end{gathered}
$$

where $t_{m n}^{\mathrm{so}}=-t_{n m}^{\mathrm{so}}$ and $t_{m n}^{B}=t_{n m}^{B}$. This Hamiltonian is the central result of this paper. The Rashba SO interaction causes two effects: (i) It gives rise to an extra phase factor $-s k_{R} x_{\beta}$ in the hopping matrix element between the leads and the QD. Note that this phase factor is dependent on the electronic spin $s$, and it is essentially different from the usual phase factor due to a magnetic flux which is independent of $s$. (ii) The Rashba SO interaction causes an interlevel spin-flip term with the strength $t_{m n}^{\mathrm{so}}$, and it cannot cause intralevel spin flips. The time-reversal invariance is maintained by the SO interaction, which is essentially different from the effect of an external magnetic field.

\section{EXAMPLE: TRANSPORT PROPERTIES OF AN $A B$ RING WITH RASHBA SO INTERACTION}

As an example of applying the second quantized Hamiltonian Eqs. (34)-(37), we now investigate the quantumtransport properties of a modified $A B$ ring (shown in Fig. 5). A QD sits on one arm of the ring and a Rashba SO interaction exists inside the QD. No Rashba interaction exists on the other arm of the ring. The ring is connected to the outside world by two normal metal leads. $A B$ rings with an embedded QD have been studied in many previous works. ${ }^{40-43}$ Some interesting phenomena, such as, for example, Fano 
resonance, ${ }^{41-43}$ have been discovered in such a device. The effect of the Rashba interaction has not been studied so far, and we have found that it leads to interesting transport behavior. In particular, a substantial spin-polarized current or conductance is induced by a combined effect of the Rashba SO interaction and a magnetic flux $\phi$ threading through the $A B$ ring. The direction of the spin polarization and its strength are easily controllable by $\phi$ or by a gate voltage.

The Hamiltonian of our $A B$ ring (Fig. 5) can be written using various pieces of the general Hamiltonian [Eqs. (34)-(37)],

$$
\begin{aligned}
H= & \sum_{k, s, \beta(\beta=L, R)} \epsilon_{\beta k} a_{\beta k s}^{\dagger} a_{\beta k s}+\sum_{s} \epsilon_{d} d_{s}^{\dagger} d_{s}+U d_{\uparrow}^{\dagger} d_{\uparrow} d_{\downarrow}^{\dagger} d_{\downarrow} \\
& +\sum_{k, s} t_{L R}\left[a_{L k s}^{\dagger} a_{R k s}+a_{R k s}^{\dagger} a_{L k s}\right]+\sum_{k, s}\left[t_{L d} a_{L k s}^{\dagger} d_{s}\right. \\
& \left.+t_{R d} e^{-i s k_{R} L} e^{i \phi} a_{R k s}^{\dagger} d_{s}\right]+ \text { H.c. }
\end{aligned}
$$

As discussed previously, the physical meaning of each term is clear. The first term describes the normal metal leads; the second term is for the QD which has a single energy level with spin-index $s$; the third term is the intradot $e$ - $e$ Coulomb interaction with a constant strength $U$; the fourth term is for the arm of the ring without the QD; and the fifth term is the coupling between the leads and the QD. Due to the Rashba SO interaction, according to Eq. (37) there is a spindependent phase factor $-s k_{R} L$ in the hopping matrix element $t_{R d}$ on the fifth term. Since we only consider one level in the QD, the interlevel spin-flip term of Eq. (35) does not appear here. This is equivalent to neglecting the intersubband mixing as in some previous works. ${ }^{8}$ We emphasize that both the $e-e$ Coulomb interaction and the Rashba SO interaction are considered, which is different from previous studies of the Rashba SO interaction where $e-e$ interaction was neglected. Indeed, our second-quantized Hamiltonian in the spectral space [Eqs. (34)-(37)] allows us to consider both effects together. Finally, the magnetic flux $\Phi$ threading the $A B$ ring gives rise to a familiar spin-independent phase factor $\phi$ $=2 \pi \Phi / \Phi_{0}$ in the matrix element $t_{R d}$.

The quantum-transport problem described by Hamiltonian (38) can be solved by standard many-body techniques. In the following we calculate the charge current using the standard Keldysh nonequilibrium Green's function method. Following Ref. 23, the charge current flowing from the left lead into the $A B$ ring, contributed by spin-up or spin-down electrons, can be derived as

$$
I_{s}=\frac{2 e}{\hbar} \int \frac{d \omega}{2 \pi} \operatorname{Re}\left[t_{L d} G_{d L s}^{<}(\omega)+t_{L R} G_{R L s}^{<}(\omega)\right],
$$

where the Keldysh Green's function $G^{<}(\omega)$ is the Fourier transform of $G^{<}(t)$, and its definition is

$$
\begin{gathered}
G_{\beta \beta^{\prime} s}^{<}(t) \equiv i\left\langle\sum_{k^{\prime}} a_{k^{\prime} \beta^{\prime} s}^{\dagger}(0) \sum_{k} a_{k \beta s}(t)\right\rangle, \\
G_{\beta d s}^{<}(t) \equiv i\left\langle d_{s}^{\dagger}(0) \sum_{k} a_{k \beta s}(t)\right\rangle,
\end{gathered}
$$

$$
G_{d d s}^{<}(t) \equiv i\left\langle d_{s}^{\dagger}(0) d_{s}(t)\right\rangle .
$$

To solve $G^{<}$, we first calculate the retarded Green functions $\mathbf{G}_{s}^{r}$ using the Dyson equation,

$$
\mathbf{G}_{s}^{r}=\mathbf{g}_{s}^{r}+\mathbf{g}_{s}^{r} \boldsymbol{\Sigma}_{s}^{r} \mathbf{G}_{s}^{r},
$$

and the Green's function $\mathbf{G}_{s}^{r}$ is a $3 \times 3$ matrix defined as

$$
\mathbf{G}_{s}^{r} \equiv\left(\begin{array}{lll}
G_{L L s}^{r} & G_{L R s}^{r} & G_{L d s}^{r} \\
G_{R L s}^{r} & G_{R R s}^{r} & G_{R d s}^{r} \\
G_{d L s}^{r} & G_{d R s}^{r} & G_{d d s}^{r}
\end{array}\right) .
$$

In Eq. (41), $\mathbf{g}_{s}^{r}$ is the Green's function of the system without coupling between the leads and the QD (i.e., when $t_{L R}=t_{L d}$ $=t_{R d}=0$ ). It can be obtained exactly as

$$
\mathbf{g}_{s}^{r}(\omega) \equiv\left(\begin{array}{ccc}
-i \pi \rho & 0 & 0 \\
0 & -i \pi \rho & 0 \\
0 & 0 & g_{d d s}^{r}(\omega)
\end{array}\right),
$$

where $g_{d d s}^{r}(\omega)=\left(\omega-\epsilon_{d}-U+U n_{\bar{s}}\right) /\left[\left(\omega-\epsilon_{d}\right)\left(\omega-\epsilon_{d}-U\right)\right]$ and $n_{\bar{s}}$ is the intradot electron occupation number at state $\bar{s} . \rho$ in Eq. (43) is the density of state of the leads. The self-energy $\Sigma_{s}^{r}(\omega)$ in Eq. (41) is ${ }^{44}$

$$
\boldsymbol{\Sigma}_{s}^{r}(\omega) \equiv\left(\begin{array}{ccc}
0 & t_{L R} & t_{L d} \\
t_{L R}^{*} & 0 & \widetilde{t}_{R d s} \\
t_{L d}^{*} & \widetilde{t}_{R d s}^{*} & 0
\end{array}\right),
$$

where $\widetilde{t}_{R d s}=t_{R d} e^{-i s k_{R} L} e^{i \phi}$. Using Eqs. (43) and (44), $\mathbf{G}_{s}^{r}$ can easily be obtained by solving Dyson's Eq. (41) as $\mathbf{G}_{s}^{r}$ $=\left(\mathbf{g}_{s}^{r-1}-\boldsymbol{\Sigma}_{s}^{r}\right)^{-1}$.

After solving $\mathbf{G}_{s}^{r}(\omega)$, the Keldysh Green's function $\mathbf{G}_{s}^{<}(\omega)$ can be obtained straightforwardly from the standard Keldysh equation,

$$
\begin{aligned}
\mathbf{G}_{s}^{<} & =\left(\mathbf{1}+\mathbf{G}_{s}^{r} \mathbf{\Sigma}_{s}^{r}\right) \mathbf{g}_{s}^{<}\left(\mathbf{1}+\mathbf{\Sigma}_{s}^{a} \mathbf{G}_{s}^{a}\right)+\mathbf{G}_{s}^{r} \mathbf{\Sigma}_{s}^{<} \mathbf{G}_{s}^{a} \\
& =\mathbf{G}_{s}^{r} \mathbf{g}_{s}^{r-1} \mathbf{g}_{s}^{<} \mathbf{g}_{s}^{a-1} \mathbf{G}_{s}^{a}+\mathbf{G}_{s}^{r} \boldsymbol{\Sigma}_{s}^{<} \mathbf{G}_{s}^{a} .
\end{aligned}
$$

For our present case, $\boldsymbol{\Sigma}_{s}^{<}=0$ and $\mathbf{g}_{s}^{r-1} \mathbf{g}_{s}^{<} \mathbf{g}_{s}^{a-1}$ is diagonal, with $\mathbf{g}_{\beta \beta s}^{r-1} \mathbf{g}_{\beta \beta s}^{<} \mathbf{g}_{\beta \beta s}^{a-1}=2 i f_{\beta}(\omega) / \pi \rho(\beta=L, R) \quad$ and $\quad \mathbf{g}_{d d s}^{r-1} \mathbf{g}_{d d s}^{<} \mathbf{s}_{d d s}^{a-1}=0$, where $f_{\beta}(\omega)=1 /\left[e^{\left(\omega-\mu_{\beta}\right) / k_{B} \mathcal{T}}+1\right]$ is the Fermi distribution function in lead $\beta$. As the last step, the intradot electron occupation number $n_{s}$ needs to be solved self-consistently with the self-consistent equation $n_{s}=-i \int(d \omega / 2 \pi) G_{d d s}^{<}(\omega)$.

In the following we present the numerical results. Figure 6 shows the total linear conductance $G=\Sigma_{s}\left(d I_{s} / d V\right)$ versus the intradot-level position $\epsilon_{d}$ at zero magnetic flux $(\phi=0)$, but with different Rashba interaction strength $k_{R} L: k_{R} L=0$ (solid), $\pi / 4$ (dashed), $\pi / 2$ (dotted), $3 \pi / 4$ (dashed-dotted), and $\pi$ (dashed-dotted-dotted). The curves are dominated by two Coulomb peaks at $\epsilon_{d}=0$ and $\epsilon_{d}=-U$. When there is no Rashba SO interaction (i.e., $k_{R} L=0$, solid curve), these two peaks show a typical Fano resonance shape due to the interference of electrons passing the two arms of the $A B$ ring, in agreement with previous theoretical and experimental studies. ${ }^{40,41}$ It is interesting to discover that this Fano resonance can be strongly affected by the Rashba SO interaction. While increasing the Rashba parameter $k_{R} L$ from 0 , the Fano 


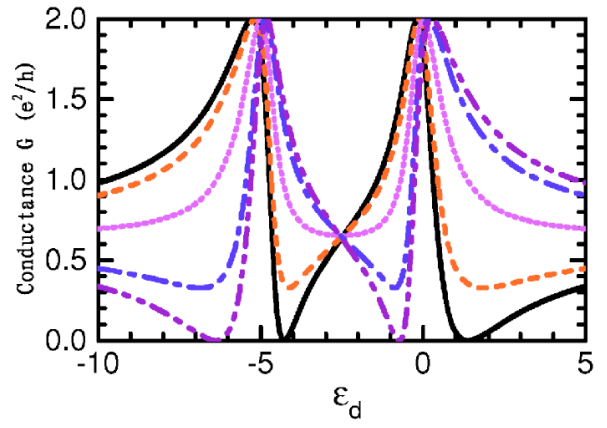

FIG. 6. (Color online) Conductance $G$ versus the intradot level $\epsilon_{d}$ for $k_{R} L=0$ (the black solid curve), $\pi / 4$ (the red dashed curve), $\pi / 2$ (the magenta dotted curve), $3 \pi / 4$ (the blue dashed-dotted curve), and $\pi$ (the purple dashed-dotted-dotted curve), respectively. Other parameters are $t_{R d}=t_{L d}=0.4, \quad t_{L R}=0.1, \quad \rho_{L}=\rho_{R}=1, \quad k_{B} \mathcal{T}$ $=0.0001, U=5$, and $\phi=0$.

resonance is decreased, and it can completely disappear at $k_{R} L=\pi / 2$ (dotted curve). While further increasing $k_{R} L$, the Fano resonance rises up again, but with an opposite Fano factor, for example, at $k_{R} L=\pi$ (dashed-dotted-dotted curve).

In order to understand these results, we investigate the interference term of total transmission probability, which is approximatively proportional to $\sim \sum_{s} \cos \left(\Delta \theta+s k_{R} L\right)$. Here $\Delta \theta$ is the phase difference of the transmission amplitude through the two arms, and it varies from 0 to $\pi / 2$ and finally to $\pi$, as $\epsilon_{d}$ is moved from $-\infty$ to 0 and finally to $\infty$. This clearly shows that the total transmission probability is indeed having a Fano asymmetric-resonance shape when $k_{R} L=0$ or $\pi$. On the other hand, it is symmetric at $k_{R} L=\pi / 2$ or $3 \pi / 2$. Hence, the Rashba SO interaction can alter the Fano resonance shape in substantial ways.

Next, in the three panels of Figs. 7 and 8, we plot conductance $G_{s}$ and spin polarization $\eta \equiv\left(G_{\uparrow}-G_{\downarrow}\right) /\left(G_{\uparrow}+G_{\downarrow}\right)$ versus magnetic flux $\phi$ for three values of $\epsilon_{d}=1,0,-1$, respectively. These values of $\epsilon_{d}$ are near the right Coulomb peak of Fig. 6. In Fig. 7, the thick curves are for $G_{\uparrow}$, and the thin curves are for $G_{\downarrow}$. The solid, dashed, and dotted curves correspond to different values of the Rashba parameter $k_{R} L$ $=0, \pi / 4$, and $\pi / 2$. Figures 7 and 8 clearly show that if either one of the two parameters $\left(\phi\right.$ and $\left.k_{R} L\right)$ vanishes, the transport current has no spin polarization, so that $\eta=0$ and $G_{\uparrow}$ $=G_{\downarrow}$. However, when both parameters are nonzero, a substantial spin-polarized conductance is found and $\eta$ can be as large as $90 \%$ for the given set of system parameters [Figs. 8(a) and 8(c)].

Importantly, in the present device the directions of spin polarization and its strength $(\eta)$ are easily controllable by varying system parameters, which are experimentally accessible. (i) By varying the magnetic flux $\phi$ : when $\phi$ is tuned from $-\pi / 2$ to $\pi / 2$ (or from $\pi / 2$ to $3 \pi / 2$ ), the polarization $\eta$ strongly varies from a large positive value to a negative value or vice versa. (ii) By varying the intradot level $\epsilon_{d}$ using a gate voltage: when $\epsilon_{d}$ is moved from one side to another side of a Coulomb peak, the polarization $\eta$ can be tuned from its largest positive value to its largest negative value or vice versa. Numerically we found that one only needs to change $\epsilon_{d}$ by a small amount to see the polarization change,

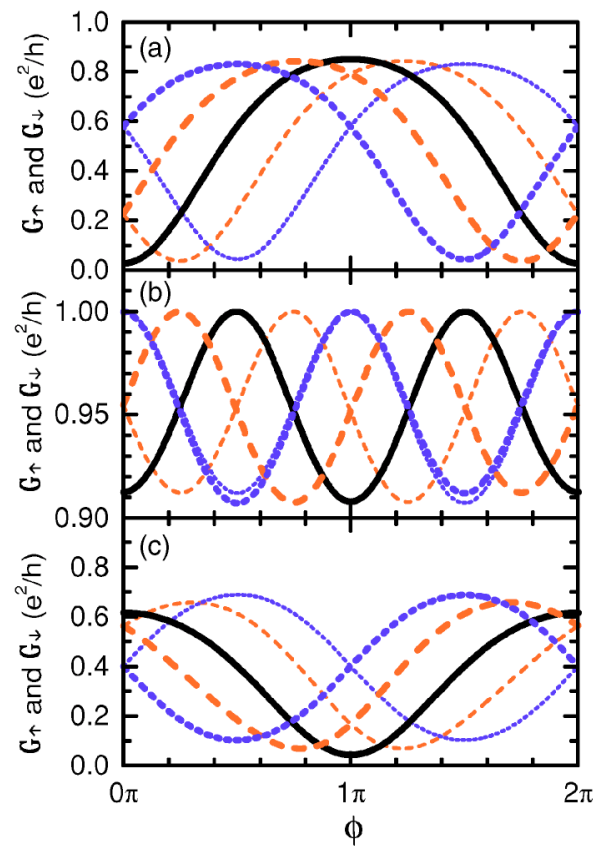

FIG. 7. (Color online) Conductance $G_{\uparrow}$ and $G_{\downarrow}$ versus magnetic flux $\phi$ for several intradot levels: (a) $\epsilon_{d}=1$; (b) $\epsilon_{d}=0$; and (c) $\epsilon_{d}=$ -1 . The solid, dashed, and dotted curves correspond to $k_{R} L$ $=0, \pi / 4$, and $\pi / 2$, respectively. The thick curves are $G_{\uparrow}$ and the thin curves are $G_{\downarrow}$. The other parameters are the same as those of Fig. 6 .

namely, a few half widths $\Gamma$ of the Coulomb peak. (The parameters used in Figs. 7 and 8 correspond to $\Gamma$ $\equiv 2 \pi \rho\left|t_{\beta d}\right|^{2} \approx 1$ ). This means that in an experiment one only

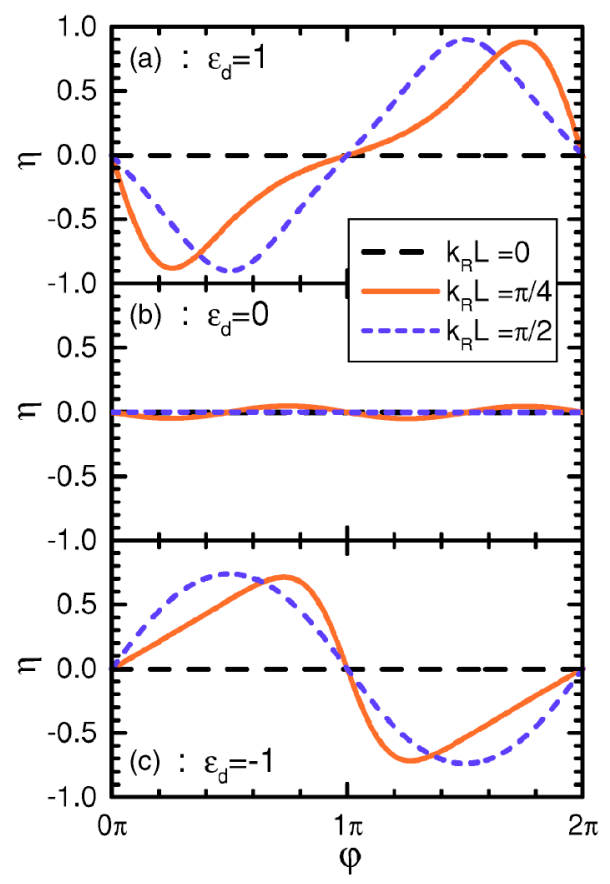

FIG. 8. (Color online) Spin polarization $\eta$ versus magnetic flux $\phi$ for $k_{R} L=0$ (dashed curve), $\pi / 4$ (solid curves), and $\pi / 2$ (dotted curve), respectively. The other parameters are the same as those of Fig. 6 . 
needs to slightly vary the gate voltage to change $\eta$ from 1 to -1 or vice versa. Furthermore, we note that when polarization $\eta$ reaches its largest value, the conductance itself is still large, e.g., $G_{\uparrow}$ or $G_{\downarrow}$ can exceed over $0.8 e^{2} / \mathrm{h}$ [see Fig. 7(a)].

Finally, we estimate if the parameter $k_{R} L$ can reach a value of $\pi / 2$ in the present experimental technology, the above theoretical predictions can be observed experimentally. Assuming the Rashba SO interaction strength $\alpha \sim 3$ $\times 10^{-11} \mathrm{eV} \mathrm{m}$, which is the reported value for some semiconductors, ${ }^{13,32,33} k_{R}=m^{*} \alpha / \hbar^{2} \approx 0.015 / \mathrm{nm}$ for $m^{*}$ $=0.036 m_{e}$. Then, if the length of the QD is the typical value $100 \mathrm{~nm}, k_{R} L \approx 1.5$. Therefore we conclude that $k_{R} L$ can reach a value $\sim \pi / 2$ or larger experimentally.

\section{CONCLUSION}

In this paper we have derived a second-quantized Hamiltonian in spectral space for a general device structure of metal-QD-metal configuration, including the spin-orbital and $e-e$ interactions. In other words, we extended the standard Anderson Hamiltonian to the case in which the central device region has a (Rashba) spin-orbital interaction. We discovered that the Rashba SO interaction causes two changes: (i) It gives rise to an extra spin-dependent phase factor $-s k_{R} x_{\beta}$ in the coupling matrix elements between the leads and the quantum dot. (ii) The Rashba SO interaction causes an interlevel spin-flip term with strength $t_{m n}^{\mathrm{so}}=-t_{n m}^{\mathrm{so}}$, and it cannot cause any intralevel spin flips.

The spectral form of the Hamiltonian is very important as it allows the analysis of many complicated quantumtransport problems involving SO and $e$-e interactions, by using the well-established many-body Green's function theoretical techniques such as the Keldysh nonequilibrium Green's function formalism. On the other hand, it would be much more difficult to carry out similar investigations using a real-space Hamiltonian, especially if $e$-e interactions are present.

As an example, we investigated the quantum-transport properties of a $A B$ ring in which a $\mathrm{QD}$ having Rashba SO and $e-e$ interactions is embedded in one arm of the ring. A substantial spin-polarized current or conductance emerges in this device due to the combined effect of a magnetic flux and the Rashba SO interaction. In particular, the direction of the spin polarization and the strength $\eta$ can be easily controlled by a number of experimentally accessible parameters.

\section{ACKNOWLEDGMENTS}

We gratefully acknowledge financial support from the Chinese Academy of Sciences and NSF-China under Grants
No. 90303016 and No. 10474125 (Q.F.S.); a RGC grant from the SAR Government of Hong Kong under Grant No. HKU 7044/04P (J.W.); the NSERC of Canada, FQRNT of Québec, and Canadian Institute of Advanced Research (H.G). Q.F.S. gratefully acknowledges Professor X.C. Xie for helpful discussions on the general physics of SO interaction.

\section{APPENDIX}

In this Appendix, we collect some general properties of the spin-orbit coupling. Although these properties should be well-known, ${ }^{13}$ we believe it is useful to put them in a form that is easily accessible. In addition, these properties hold for all spin-orbit interactions, including the Rashba SO interaction.

(i) The SO interaction Hamiltonian $H_{\text {so }}$, [Eq. (5)], satisfies the time-reversal invariance. In other words, $H_{\text {so }}$ commutes with the time-reversal operator $T=-i \hat{\sigma}_{y} K$ (where $K$ is the complex-conjugation operator). Indeed, using the Hamiltonian $H_{\mathrm{so}}$ of Eq. (5), it is easy to prove $\left[T, H_{\mathrm{so}}\right]=0$.

(ii) When a system has spin-orbit coupling, each eigenenergy level is still at least twofold degenerate, i.e., the socalled Kramer's degeneracy exists. Briefly this can be proved as follows. We start from the Hamiltonian $H$ of Eq. (3) but, setting $\mathbf{B}=\mathbf{M}(\mathbf{r})=0$ in (3), we assume $\varphi_{n}\left(\mathbf{r}_{1}, \mathbf{r}_{2}, \ldots \mathbf{r}_{N}\right)$ is an eigenstate of $H$, so that $H\left|\varphi_{n}\right\rangle=E_{n}\left|\varphi_{n}\right\rangle$. Since $H$ is timereversal invariant $(T H=H T)$, we have $H T\left|\varphi_{n}\right\rangle=T H\left|\varphi_{n}\right\rangle$ $=E_{n} T\left|\varphi_{n}\right\rangle$. Hence, state $T\left|\varphi_{n}\right\rangle$ is also an eigenstate with the same eigenenergy $E_{n}$ as that of the state $\left|\varphi_{n}\right\rangle$. Furthermore, one has $\left\langle\varphi_{n}|T| \varphi_{n}\right\rangle=\left(\varphi_{n}, T \varphi_{n}\right)=\left(T^{2} \varphi_{n}, T \varphi_{n}\right)=-\left\langle\varphi_{n}|T| \varphi_{n}\right\rangle$, hence $\left\langle\varphi_{n}|T| \varphi_{n}\right\rangle=0$. This means state $T\left|\varphi_{n}\right\rangle$ is orthogonal to $\left|\varphi_{n}\right\rangle$. Therefore, although spin is no longer a good quantum number when the SO interaction exists, the system is still at least twofold degenerate for any of its eigenstates.

(iii) At equilibrium, a spin-orbit coupling cannot induce a spontaneous spin polarization. We prove this as follows. Since the system is in equilibrium, the twofold degenerate eigenstates $\left|\varphi_{n}\right\rangle$ and $T\left|\varphi_{n}\right\rangle$ have the same occupation probability $p\left(E_{n}\right)$. Then, the average of spin polarization in an arbitrary direction $\hat{n}$ can be calculated as

$$
\begin{aligned}
\left\langle\hat{\sigma}_{\hat{n}}\right\rangle= & \sum_{n} p\left(E_{n}\right)\left[\left\langle\varphi_{n}\left|\hat{\sigma}_{\hat{n}}\right| \varphi_{n}\right\rangle+\left\langle T \varphi_{n}\left|\hat{\sigma}_{\hat{n}}\right| T \varphi_{n}\right\rangle\right] \\
& =\sum_{n} p\left(E_{n}\right)\left[\left\langle\varphi_{n}\left|\hat{\sigma}_{\hat{n}}\right| \varphi_{n}\right\rangle+\left\langle T \varphi_{n}\left|-T \hat{\sigma}_{\hat{n}}\right| \varphi_{n}\right\rangle\right] \\
& =\sum_{n} p\left(E_{n}\right)\left[\left\langle\varphi_{n}\left|\hat{\sigma}_{\hat{n}}\right| \varphi_{n}\right\rangle-\left\langle\varphi_{n}\left|\hat{\sigma}_{\hat{n}}\right| \varphi_{n}\right\rangle\right]=0 .
\end{aligned}
$$

Therefore, at equilibrium no spin-orbit coupling can induce a spontaneous spin polarization in any direction.

\footnotetext{
*Electronic address: sunqf@aphy.iphy.ac.cn

${ }^{1}$ S. A. Wolf, D. D. Awschalom, R. A. Buhrman, J. M. Daughton,

S. von Molnar, M. L. Roukes, A. Y. Chtchelkanova, and D. M.

Treger, Science 294, 1488 (2001).

${ }^{2}$ G. A. Prinz, Science 282, 1660 (1998).
}

${ }^{3}$ I. Zutic, J. Fabian, and S. Das Sarma, Rev. Mod. Phys. 76, 323 (2004).

${ }^{4}$ S. Murakami, N. Nagaosa, and S.-C. Zhang, Science 301, 1348 (2003).

${ }^{5}$ J. Sinova, D. Culcer, Q. Niu, N. A. Sinitsyn, T. Jungwirth, and A. 
H. MacDonald, Phys. Rev. Lett. 92, 126603 (2004).

${ }^{6}$ Y. K. Kato, R. C. Myers, A. C. Gossard, and D. D. Awschalom, Science 306, 1910 (2004); J. Wunderlich, B. Kastner, J. Sinova, and T. Jungwirth, Phys. Rev. Lett. 94, 047204 (2005).

${ }^{7}$ S.-Q. Shen, M. Ma, X. C. Xie, and F. C. Zhang, Phys. Rev. Lett. 92, 256603 (2004); S.-Q. Shen, Y.-J. Bao, M. Ma, X. C. Xie, and F. C. Zhang, cond-mat/0410169 (unpublished).

${ }^{8}$ S. Datta and B. Das, Appl. Phys. Lett. 56, 665 (1990).

${ }^{9}$ D. Culcer, J. Sinova, N. A. Sinitsyn, T. Jungwirth, A. H. MacDonald, and Q. Niu, Phys. Rev. Lett. 93, 046602 (2004).

${ }^{10}$ E. I. Eashba and AI. L. Efros, Phys. Rev. Lett. 91, 126405 (2003).

${ }^{11}$ L. P. Rokhinson, V. Larkina, Y. B. Lyanda-Geller, L. N. Pfeiffer, and K. W. West, Phys. Rev. Lett. 93, 146601 (2004).

${ }^{12}$ L. Hu, J. Gao, and S.-Q. Shen, Phys. Rev. B 68, 115302 (2003).

${ }^{13}$ F. Mireles and G. Kirczenow, Phys. Rev. B 64, 024426 (2001).

${ }^{14}$ M. Cahay and S. Bandyopadhyay, Phys. Rev. B 68, 115316 (2003).

${ }^{15}$ M. Larsen, A. M. Lunde, and K. Flensberg, Phys. Rev. B 66, 033304 (2002).

${ }^{16}$ T. Matsuyama, C.-M. Hu, D. Grundler, G. Meier, and U. Merkt, Phys. Rev. B 65, 155322 (2002).

${ }^{17}$ X. F. Wang and P. Vasilopoulos, Appl. Phys. Lett. 83, 940 (2003); Phys. Rev. B 67, 085313 (2003).

${ }^{18}$ J. Wang, H. B. Sun, and D. Y. Xing, Phys. Rev. B 69, 085304 (2004).

${ }^{19}$ Jorgen Rammer, Quantum Transport Theory (Perseus Books, Massachusetts, 1998).

${ }^{20}$ Gerald D. Mahan, Many-Particle Physics (Plenum Press, New York, 1981)

${ }^{21}$ A. C. Hewson, The Kondo Problem to Heavy Fermions (Cambridge University Press, Cambridge, 1993).

${ }^{22}$ K. C. Chou, Z. B. Su, B. L. Hao, and L. Yu, Phys. Rep. 118, 1 (1985).

${ }^{23}$ Y. Meir and N. S. Wingreen, Phys. Rev. Lett. 68, 2512 (1992); A.-P. Jauho, N. S. Wingreen, and Y. Meir, Phys. Rev. B 50, 5528 (1994).

${ }^{24}$ J. D. Bjorken and S. D. Drell, Relativistic Quantum Mechanics (McGraw-Hill, New York, 1965).

25 In order to simplify notation, the Bohr magneton $\mu_{B}$ in Eq. (3) has been absorbed into $\mathbf{M}(\mathbf{r})$ and $\mathbf{B}(\mathbf{r})$.

${ }^{26}$ E. I. Rashba, Fiz. Tverd. Tela (Leningrad) 2, 1224 (1960) [Sov. Phys. Solid State 2, 1109 (1960)]

${ }^{27}$ Y. A. Bychkov and E. I. Rashba, J. Phys. C 17, 6039 (1984).

${ }^{28}$ Q.-F. Sun, H. Guo, and J. Wang, Phys. Rev. B 69, 054409 (2004).

${ }^{29}$ Since the electron is accelerated under the influence of the electric field $\mathbf{E}$, its spin has a Thomas procession due to the relativistic effect, so that the interactive potential energy is then decreased by a factor 2. See J. D. Jackson, Classical Electrodynamics, 2nd ed. (John Wiley \& Sons, Inc., New York, 1975), pp. 542-546.

${ }^{30}$ Note that in principle $\alpha$ can depend on coordinate $x$, for instance when $\alpha$ varies sharply at the metal-QD interface. Therefore usually $\mathbf{p} \alpha(x) \neq \alpha(x) \mathbf{p}$. Thereby the two terms in Eq. (7) [or Eq. (13)] are not completely the same.

${ }^{31}$ J. Nitta, T. Akazaki, H. Takayanagi, and T. Enoki, Phys. Rev. Lett. 78, 1335 (1997).

${ }^{32}$ T. Matsuyama, R. Kursten, C. Meissner, and U. Merkt, Phys. Rev. B 61, 15588 (2000).

${ }^{33}$ D. Grundler, Phys. Rev. Lett. 84, 6074 (2000)

${ }^{34}$ J. P. Heida, B. J. van Wees, J. J. Kuipers, T. M. Klapwijk, and G. Borghs, Phys. Rev. B 57, 11911 (1998).

${ }^{35}$ N. Sergueev, Q.-F. Sun, H. Guo, B. G. Wang, and J. Wang, Phys. Rev. B 65, 165303 (2002).

${ }^{36}$ U. Zulicke and C. Schroll, Phys. Rev. Lett. 88, 029701 (2002).

37 Because $\alpha=0$ in the metal regions $\left(x<x_{L}\right.$ and $\left.x>x_{R}\right)$ and $\alpha$ $\neq 0$ in the QD region $\left(x_{L}<x<x_{R}\right)$, there is a $\delta$ function emerging from the term $\hat{\sigma}_{z} p_{x} \alpha(x)$.

${ }^{38}$ L. W. Molenkamp, G. Schmidt, and G. E. W. Bauer, Phys. Rev. B 64, 121202 (2001).

39 If assuming that a single-particle operator $\hat{X}(\mathbf{r})$ has the timereversal invariance, i.e., $[\hat{X}, T]=0$, its matrix elements of the second quantization have the properties $X_{n \uparrow, m \uparrow}=X_{n \downarrow, m \downarrow}^{*}$ and $X_{n \uparrow, m \downarrow}=-X_{n \downarrow, m \uparrow}^{*}, \quad$ because $\quad X_{n \uparrow, m \uparrow}=\langle n \uparrow|\hat{X}| m \uparrow\rangle$ $=\left\langle e^{i c} T n \downarrow|\hat{X}| e^{i c} T m \downarrow\right\rangle=\langle T n \downarrow \mid T \hat{X} m \downarrow\rangle=\langle\hat{X} m \downarrow \mid n \downarrow\rangle=\langle n \downarrow|\hat{X}| m \downarrow\rangle^{*}$ $=X_{n \downarrow, m \downarrow}^{*}, \quad$ and $\quad X_{n \uparrow, m \downarrow}=\langle n \uparrow|\hat{X}| m \downarrow\rangle=\langle T n \downarrow|\hat{X}(-) T| m \uparrow\rangle=$ $-\langle\hat{X} m \uparrow \mid n \downarrow\rangle=-X_{n \downarrow, m \uparrow}^{*} . \quad$ Here $\quad|n \uparrow\rangle=e^{i c} T|n \downarrow\rangle \quad$ and $\quad|n \downarrow\rangle=$ $-e^{i c} T|n \uparrow\rangle$ with $c$ as an arbitrary real constant. On the other hand, if the matrix elements of an operator $\hat{X}$ have the properties $X_{n \uparrow, m \uparrow}=X_{n \downarrow, m \downarrow}^{*}$ and $X_{n \uparrow, m \downarrow}=-X_{n \downarrow, m \uparrow}^{*}$, it can easily be proven that this operator $\hat{X}$ has the time-reversal invariance, $[\hat{X}, T]=0$.

${ }^{40}$ A. Yacoby, M. Heiblum, D. Mahalu, and H. Shtrikman, Phys. Rev. Lett. 74, 4047 (1995); R. Schuster, E. Buks, M. Heiblum, D. Mahalu, V. Umansky, and H. Shtrikman, Nature (London) 385, 417 (1997); G. Hackenbroich, Phys. Rep. 343, 463 (2001).

${ }^{41}$ C.-M. Ryu and S. Y. Cho, Phys. Rev. B 58, 3572 (1998).

${ }^{42}$ Z. Ma, Y. Zhu, X.-Q. Li, T.-H. Lin, and Z.-B. Su, Phys. Rev. B 69, 045302 (2004).

${ }^{43}$ Z. Y. Zeng, F. Claro, and A. Perez, Phys. Rev. B 65, 085308 (2002).

${ }^{44}$ Note that this method of solving $\mathbf{G}^{r}$ is not exact. It is equivalent to neglecting higher-order self-energy terms. This is a good approximation for temperatures higher than the Kondo temperature. 